ORIGINAL ARTICLE

\title{
Prospective evaluation of non-radiologist performed emergency abdominal ultrasound for haemoperitoneum
}

\author{
A Brooks, B Davies, M Smethhurst, J Connolly
}

Emerg Med J 2004;21:e5 (http://www.emjonline.com/cgi/content/full/21/5/e5). doi: 10.1136/emj.2003.006932

Objectives: To evaluate non-radiologist performed emergency ultrasound for the detection of haemoperitoneum after abdominal trauma in a British accident and emergency department.

Methods: Focused assessment with sonography for trauma (FAST) was performed during the primary survey on adult patients triaged to the resuscitation room with suspected abdominal injury over a 12 month period. All investigations were performed by one of three non-radiologists trained in FAST. The ultrasound findings were compared against the investigation of choice of the attending surgeon/accident and emergency physician. The patients were followed up for clinically significant events until hospital discharge or death.

See end of article for authors' affiliations

.....................

Correspondence to: Mr A Brooks, Department of Surgery, Queens Medical Centre, University Hospital, Nottingham NG7 2UH, UK; adambrooks@ doctors.org.uk

Accepted for publication 19 March 2003

Results: One hundred patients who had sustained blunt abdominal trauma, were evaluated by FAST. Nine true positive scans were detected and confirmed by computed tomography, diagnostic peritoneal lavage, or laparotomy. There was one false positive in this group, giving a sensitivity of $100 \%$, specificity $99 \%$, and positive predictive value of $90 \%$. Ten patients with penetrating injuries were evaluated with a sensitivity and specificity for FAST of $33 \%$ and $86 \%$ respectively.

Conclusions: Emergency torso ultrasound for the detection of haemoperitoneum can be successfully performed by trained non-radiologists within a British accident and emergency system. It is an accurate and rapid investigation for blunt trauma, but the results should be interpreted with caution in penetrating injury.

$\mathrm{F}^{\mathrm{s}}$ ocused assessment with sonography for trauma (FAST) is a technique initially developed by Rozycki et $a l^{12}$ for the ultrasound assessment of abdominal trauma. The system is based on the principle that haemoperitoneum collects primarily in three dependent regions: perihepatic, perisplenic, and pelvis. The procedure uses the focused sequential ultrasound assessment of these regions for blood, in addition a pericardial view is obtained to assess for cardiac tamponade. The technique was designed principally for nonradiologists-emergency physicians and surgeons involved in the resuscitation of trauma patients. North American studies have shown it to be an effective and sensitive technique for the detection of blunt abdominal trauma ${ }^{3}$ and equally accurate in the hands of non-radiologists and radiologists alike. ${ }^{4}$ These studies have predominately been undertaken in busy specialist American trauma centres with a consistently high flow of significantly injured patients. The volume of severe trauma differs considerably in the United Kingdom, where an estimated 10000 patients suffer multiple injuries per year ${ }^{5}$ and most of the 230 acute accident and emergency departments will admit on average one multiply injured patient per 1000 emergency cases. ${ }^{5}$ Data on the value of nonradiologist performed FAST within the confines of a British accident and emergency department are to date limited. ${ }^{6}$

The aim of this study is to evaluate non-radiologist performed emergency torso ultrasound against established investigations for the detection of intra-abdominal blood in patients with abdominal trauma in a British accident and emergency department.

\section{METHODS}

Adult patients triaged to the resuscitation room of Queens Medical Centre (QMC), Nottingham with multiple trauma or suspected abdominal injury over a 12 month period underwent ultrasound investigation using the FAST technique. FAST was undertaken during the primary survey by one of three investigators-an accident and emergency consultant and a staff grade and a general surgical specialist registrar. Horizontal organisation of the trauma team ensured that the ultrasound investigation did not delay the resuscitation. FAST was performed using a Sonosite 180 hand carried ultrasound system with a 4-2 MHz transducer. The use of the ultrasound system was dedicated to the study and was available within accident and emergency 24 hours a day. The images were stored for immediate review and the findings of the emergency physician sonographer recorded and declared to the trauma team as required by the ethics committee.

The ultrasound results were subsequently compared against the investigation of choice of the attending surgeon/ accident and emergency physician-computed tomography (CT), diagnostic peritoneal lavage (DPL), laparotomy, or clinical observation. All clinical decisions were taken based on information collected from these investigations. Physiological and injury data were recorded at the time of the patients' arrival and their clinical course followed up for significant events related to potential abdominal injuries until hospital discharge or death.

Ethical approval for the study was granted by the Queens Medical Centre ethics committee reference GS040102.

\section{RESULTS}

One hundred and ten patients were evaluated by FAST after abdominal trauma. Ten of these patients had sustained penetrating injury and were analysed separately; all were stab wounds to the abdominal, thoracoabdominal region, or loin. All FAST scans were completed within four minutes.

Figure 1 shows the mechanisms of injury sustained in the 100 blunt trauma patients. The mean injury severity score

Abbreviations: CT, computed tomography; FAST, focused assessment with sonography for trauma; DPL, diagnostic peritoneal lavage; RTS, revised trauma score; ISS, injury severity score 

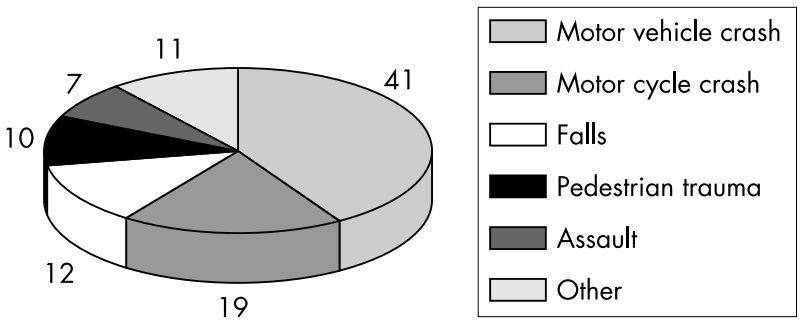

Figure 1 Mechanism of injury.

(ISS) of the blunt trauma victims was 13.95 and revised trauma score (RTS) 6.96. Figure 2 shows the results of the FAST scan and the investigations used to confirm them. Three patients who had a positive FAST result confirmed by CT progressed to laparotomy for abdominal bleeding. One false positive scan was detected on reviewing the recorded images while the secondary survey was progressing. The "fluid" that had been reported was in a "non-classical" position; a DPL was performed, which was negative. Twenty three patients were observed until hospital discharge after a negative FAST (median in patient observation 5 days, range 1-21 days), none developed abdominal complications. Five patients died from their injuries all had FAST results consistent with the findings at surgery/postmortem examination. The sensitivity of FAST for the evaluation of blunt abdominal trauma was $100 \%$ and specificity $99 \%$, with a positive predictive value of $90 \%$.

Ten patients sustained penetrating injury from stab wounds and the results are shown in figure 3. The mean ISS and RTS values were 7 and 7.55 respectively. In the first false negative scan the patient remained cardiovascularly stable with minimal abdominal signs for two hours until theatre. At laparoscopy the peritoneum had been breached and there was $200 \mathrm{ml}$ of blood in the abdomen from a bleeding superior epigastric artery, there were no visceral or intra-abdominal injuries. The second false negative scan occurred in a patient with stab wounds to the anterior abdominal wall. Laparotomy revealed isolated wounds to the stomach and jejunum with minimal intra-peritoneal fluid. The sensitivity of FAST in penetrating trauma was 33\%, specificity $86 \%$.

Five scans were technically incomplete; in two pericardial views were not obtained although abdominal windows were satisfactory, in three surgical emphysema from associated chest trauma obscured views of the left upper quadrant. CT (two patients) and DPL (one patient) were used to confirm
FAST, all were negative. None of the false negative or positive scans led to mortality or morbidity.

\section{DISCUSSION}

FAST has previously been shown to be a valuable investigation for the assessment of blunt abdominal trauma in large series from several North America trauma centres. ${ }^{1{ }^{3}}$ Results from these institutions report a sensitivity of $80 \%-88 \%$ and specificity of $90 \%-99 \%$ for non-radiologists using the technique. Other studies have shown that the ability to detect haemoperitoneum using the FAST technique is equally accurate in the hands of non-radiologists and radiologists alike. $^{48}$

Queens Medical Centre, Nottingham has one of the largest accident and emergency departments in the UK and more than 200 resuscitations are undertaken for severely injured patients annually. The number of patients in the study, although small by international standards, is representative of the workload over the study period and is likely to be greater than many other institutions in the UK.

This study has evaluated two aspects of the use of FAST in the assessment of trauma patients in a British accident and emergency department. The technique has firstly been evaluated against established investigations in a cohort of British trauma patients. Secondly, the ability of trained nonradiologists from different specialties to perform FAST with acceptable accuracy has been determined and can therefore be compared against international results.

FAST has previously been compared favourably to CT and DPL in the investigation of blunt abdominal injury. ${ }^{9}{ }^{10}$ Each technique has inherent advantages and disadvantages. CT is currently accepted as the gold standard for the investigation of the trauma patient. In addition to providing evidence of bleeding it gives detailed anatomical information of injuries. It is associated with a sensitivity of $88 \%$ and specificity of $98 \%{ }^{11}$ However, it is unsuitable for the unstable multiply injured patient. Although a sensitive investigation for abdominal bleeding ${ }^{12}$ and previously the gold standard for the abdominal investigation of the unstable trauma patient, the invasive nature of DPL and the high incidence of nontherapeutic laparotomy after a positive result, means it has largely fallen from favour in many institutions. Previous work from our institution has shown practical difficulties undertaking DPL in the UK. ${ }^{13}$ FAST is a non-invasive, rapidly performed bedside investigation ideally suited to the initial assessment of the trauma patient in the resuscitation room. However, the limitations of a negative FAST examination have come to be recognised ${ }^{14}{ }^{15}$ and it has been suggested that

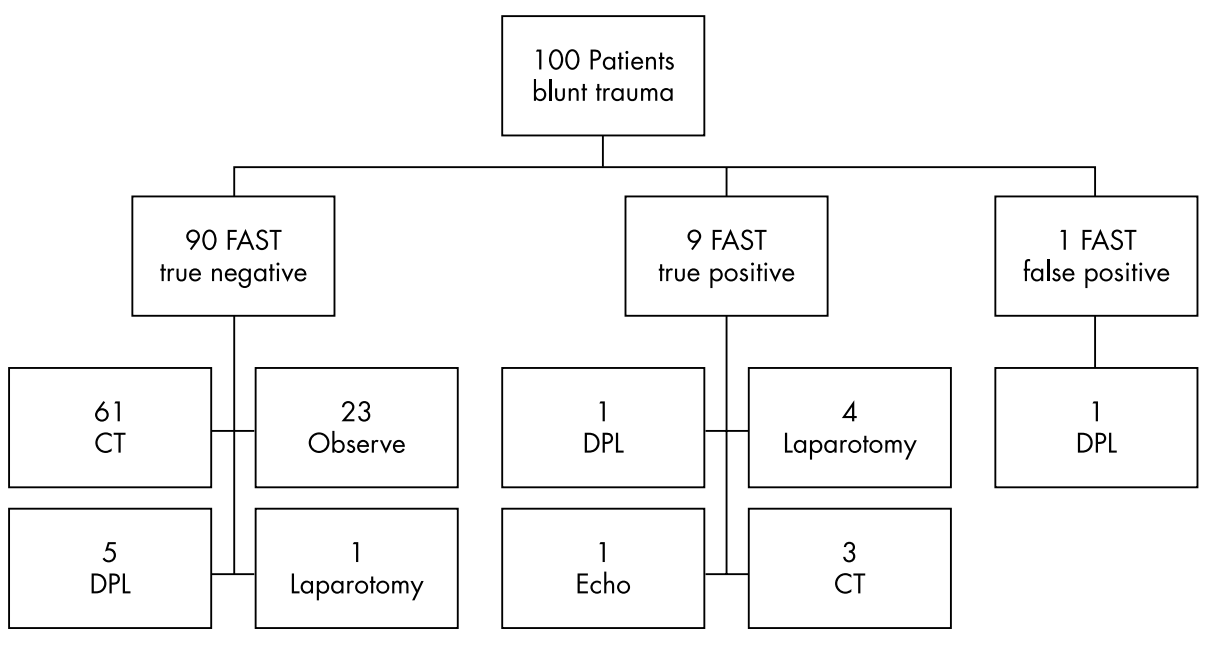

Figure 2 FAST results and investigations to confirm the FAST findings for blunt trauma. 


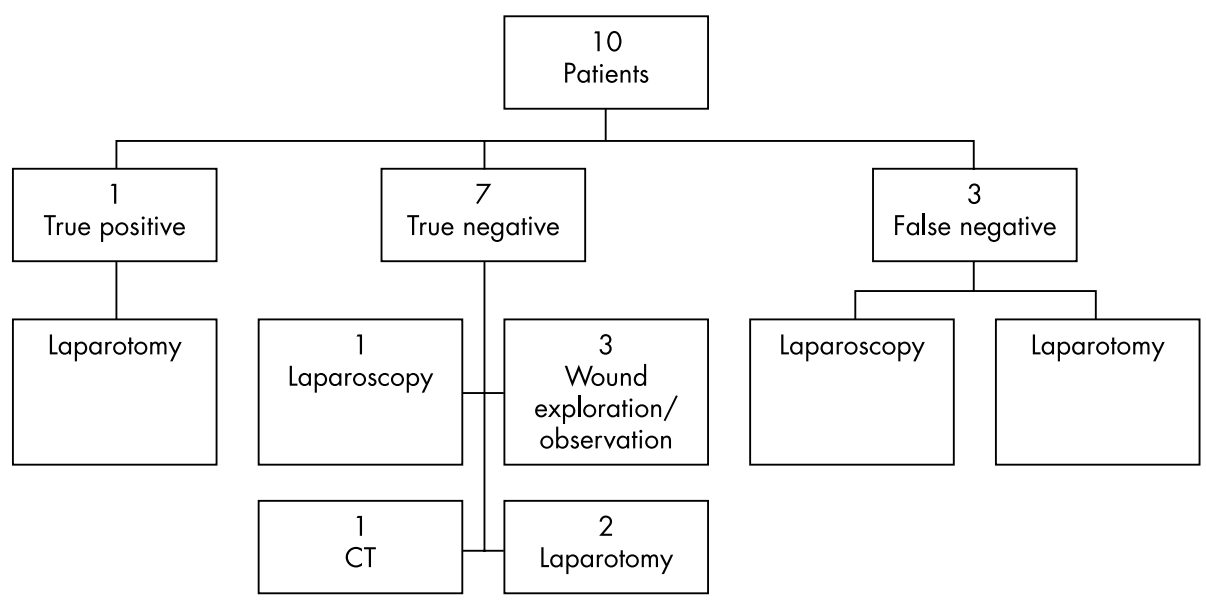

Figure 3 FAST results and investigations for penetrating trauma.

a negative FAST should be repeated at an interval of six hours ${ }^{16}$ or an alternative modality used. Repeat FAST was not performed on the patients with a negative scan in this series as most proceeded to CT scan or an alternate investigation. While it would have been ideal to repeat the FAST in the group of patients who were simply observed clinically, practical difficulties precluded this. None of this group developed abdominal related complications or underwent abdominal imaging during their inpatient admission.

The results of FAST in blunt trauma from this study are accurate compared with evaluation with CT, DPL, or clinical observation. The comparatively small number of patients with haemoperitoneum in the series, although representative of the incidence at our centre, may account for the high sensitivity in this study. FAST cannot replace the precise anatomical information provided by CT scan. It is extremely valuable in the initial assessment of the unstable injured patient and a useful adjunct to CT scan in stable patients.

Table 1 shows that this study compares favourably with international work evaluating the ability of radiologists and non-radiologists to detect haemoperitoneum in blunt trauma using FAST.

The limited number of patients with penetrating injuries in our study precludes meaningful conclusions. Previous studies looking at FAST in penetrating trauma have to date been disappointing. Kahdi ${ }^{17}$ evaluated 75 patients with penetrating injuries and reported 22 false negative scans and a sensitivity of only $46 \%$. The sensitivity of FAST in our study (50\%) is equivalent to Kahdi's work. Caution must be used evaluating penetrating injury with FAST. While positive studies are a strong predictor of injury, negative scans must be augmented by additional investigations. At QMC it is policy to evaluate equivocal abdominal and thoracoabdominal stab wounds with laparoscopy and posterior wounds with contrast enhanced CT.

All the FAST scans in this series were completed within a four minute time period. The horizontal organisation of the trauma team allowed FAST to proceed without delay or disruption in the flow of the resuscitation. No attempt was

Table 1 Comparison for blunt trauma

\begin{tabular}{|c|c|c|c|c|c|}
\hline & \multirow{2}{*}{$\frac{\text { QMC study }}{\text { NR }}$} & \multicolumn{2}{|c|}{ Rozycki $^{4}$} & \multicolumn{2}{|c|}{ Buzzas $^{8}$} \\
\hline & & NR & $\mathbf{R}$ & NR & $\mathbf{R}$ \\
\hline Sensitivity (\%) & 100 & 93.4 & 90.8 & 79.5 & 79.5 \\
\hline Specificity (\%) & 99 & 98.7 & 99.2 & 97.5 & 99.3 \\
\hline
\end{tabular}

made to differentiate the length of time taken for positive or negative FAST in this study. Boulanger has previously reported that in cardiovascularly unstable patients FAST will reveal intra-abdominal blood within 20 seconds. ${ }^{18}$ FAST, if used as the primary investigative modality, provides a rapid answer to abdominal evaluation during the circulation assessment.

The issue of training for non-radiologists in trauma ultrasound remains contentious and has not been specifically addressed by this study. The three primary investigators had each undergone a formal one day training package (American College of Surgeons ultrasound in an emergency setting course or a United Kingdom equivalent) followed by a minimum of 15 FAST scans performed under close supervision before participation in the study. This is in line with the American College of Surgeons recommendations of an eight hour trauma US course, which has been shown to be adequate for the successful performance of the technique, ${ }^{19}$ followed by a minimum of 10 proctored scans. Review of all the recorded images by the most experienced investigator and regular feedback was used as an effective quality assurance programme.

Emergency torso ultrasound is an accurate and rapid investigation for the detection of haemoperitoneum in blunt trauma and can be successfully performed by trained nonradiologists in the UK. We recommend that FAST should be adopted as the initial investigation to augment the clinical assessment of abdominal trauma in the UK. However, the limitations of a negative FAST must be recognised and the results should be interpreted with caution in penetrating injury.

\section{Authors' affiliations}

A Brooks, B Davies, Department of Surgery, Queens Medical Centre, University Hospital, Nottingham, UK

M Smethhurst, J Connolly, Department of Accident and Emergency, Queens Medical Centre

Conflicts of interest: none declared.

\section{REFERENCES}

1 Rozycki GS, Ochsner MG, Jaffin JH, et al. Prospective evaluation of surgeons' use of ultrasound in the evaluation of trauma patients. J Trauma 1993;34:516-26.

2 Rozycki GS, Ochsner MG, Schmidt JA, et al. A prospective study of surgeonperformed ultrasound as the primary adjuvant modality for injured patient assessment. J Trauma 1995;39:492-500.

3 Dolich MO, McKenney MG, Varela JE, et al. 2576 ultrasounds for blunt abdominal trauma. J Trauma 2001;50:108-12.

4 Rozycki GS, Shackford SR. Trauma ultrasound for surgeons. In: Staren ED, ed. Ultrasound for the Surgeon. New York: Lippincott-Raven, 1997:120-35. 
5 Burdett-Smith P, Airey M, Franks AJ. Improvement in trauma survival in Leeds. Injury 1995;26:455-8.

6 Sloan JP, Lalanda M, Brenchley J, et al. Developing the role of emergency medicine ultrasonography. The Leeds experience. Emerg Med J 2002; 19:A63

7 McKenney MG, Martin L, Lentz K, et al. 1000 consecutive ultrasounds for blunt abdominal trauma. J Trauma 1996;40:607-12.

8 Buzzas GR, Kern SJ, Smith SR, et al. A comparison of sonographic examinations for trauma performed by surgeons and radiologists. J Trauma 1998;44:604-8.

9 McKenney M, Lentz K, Nunez D, et al. Can ultrasound replace diagnostic peritoneal lavage in the assessment of blunt trauma. J Trauma 1994;37:339-441.

10 Boulanger BR, McLellan BA, Brenneman FD, et al. Prospective evidence of superiority of a sonography based algorithm in the assessment of blunt abdominal injury. J Trauma 1999;47:632-7.

11 Catre MG. Diagnositic peritoneal lavage versus abdominal computed tomography in blunt abdominal trauma: a review of prospective studes. Can J Surg 1995;38:117-22.

12 Nagy KK, Roberts RR, Joseph KT, et al. Experience with over 2500 diagnostic peritoneal lavages. Injury 2000;31:479-82.
13 Maxwell-Armstrong C, Rooks A, Field M, et al. Diagnostic peritoneal lavage. Should trauma guidelines be revised? Emerg Med J 2002; 19:524-5.

14 Chiu WC, Cushing BM, Rodriguez A, et al. Abdominal injuries without haemoperitoneum: a potential limitation of focused abdominal sonography for trauma. J Trauma 1997;42:617-25.

15 Ballard RB, Rozycki GS, Newman PG, et al. An algorithm to reduce the incidence of false-negative FAST examinations in patients at high risk for occult injury. Focused assessment for the sonographic examination of the trauma patient. J Am Coll Surg 1999;189:145-50.

16 Scalea TM, Rodriguez A, Chiu WC, et al. Focused assessment with sonograpy for trauma (FAST): Results from an international consensus conference. $J$ Trauma 1999;46:444-72.

17 Udobi KF, Rodriguez A, Chiu WC, et al. Role of ultrasonography in penetrating abdominal trauma: a prospective clinical study. J Trauma 2001;50:475-9.

18 Boulanger BR, Brenneman FD, McLellan BA, et al. A prospective study of emergent abdominal sonography after blunt abdominal trauma. J Trauma 1995;39:325-30.

19 Thomas B, Falcone RE, Vasquez D, et al. Ultrasound evaluation of blunt abdominal trauma: program implementation, initial experience, and learning curve. J Trauma 1997; 42:384-90. 